\title{
Catalogue of far-infrared loops in the Galaxy ${ }^{\star}$
}

\author{
V. Könyves ${ }^{1}$, Cs. Kiss ${ }^{2}$, A. Moór ${ }^{2}$, Z. T. Kiss ${ }^{3}$, and L. V. Tóth ${ }^{1}$ \\ 1 Astronomy Department, Eötvös Loránd University, PO Box 32, 1518 Budapest, Hungary \\ e-mail: [v.konyves; l.v.toth] aastro.elte.hu \\ 2 Konkoly Observatory of the Hungarian Academy of Sciences, PO Box 67, 1525 Budapest, Hungary \\ e-mail: [pkisscs;moor] @konkoly.hu \\ 3 Baja Astronomical Observatory of Bács-Kiskun County, PO Box 766, 6500 Baja, Hungary \\ e-mail: kissz@alcyone.bajaobs.hu
}

Received 14 April 2006 / Accepted 12 October 2006

ABSTRACT

\begin{abstract}
Aims. An all-sky survey of loop- and arc-like intensity enhancements has been performed to investigate the large-scale structure of the diffuse far-infrared emission.

Methods. We used maps made of 60 and $100 \mu$ m processed IRAS data (Sky Survey Atlas and dust infrared emission maps) to identify large-scale structures: loops, arcs, or cavities, in the far-infrared emission in the Galaxy. Distances were attributed to a subsample of loops using associated objects.

Results. We identified 462 far-infrared loops, analysed their individual FIR properties and their distribution. This data forms the Catalogue of Far-Infrared Loops in the Galaxy. We obtained observational estimates of $f_{\text {in }} \approx 30 \%$ and $f_{\text {out }} \approx 5 \%$ for the hot gas volume filling factor of the inward and outward Galactic neighbourhood of the Solar System. We obtained a slope of the power-law size luminosity function $\beta=1.37$ for low Galactic latitudes in the outer Milky Way.

Conclusions. Deviations in the celestial distribution of far-infrared loops clearly indicate that violent events frequently overwrite the structure of the interstellar matter in the inner Galaxy. Our objects trace out the spiral arm structure of the Galaxy in the neighbourhood of the Sun and their distribution clearly suggests that there is an efficient process that can generate loop-like features at high Galactic latitudes. Power-law indices of size luminosity distributions suggest that the structure of the ISM is ruled by supernovae and stellar winds at low Galactic latitudes, while it is governed by supersonic turbulence above the Galactic plane.
\end{abstract}

Key words. catalogs - ISM: bubbles - Galaxy: structure

\section{Introduction}

The large-scale structure of the cold interstellar matter can be significantly affected by violent events. This structure - which is diverse with the complex distribution of shells, cavities, filaments, arcs, and loops - is often referred to as the "Cosmic Bubble Bath" (Brand \& Zealey 1975). The evolution of bubbles and superbubbles produced by supernova explosions and stellar winds of associations are the primary processes that determine the structure and energetics of all components of the diffuse interstellar medium. Studying supernova (SN) explosions in a uniform medium, Cox \& Smith (1974) pointed out that if the Galactic SN rate is "sufficient" it can produce the "swiss cheese morphology" of the cold diffuse ISM with hot coronal gas inside the bubbles. This was built into the model by McKee \& Ostriker (1977), where SN explosions in the cloudy ISM produce a threephase medium. In this scenario the next generation of stars are born in the compressed medium of the bubble walls (propagating star formation, see Blaauw 1991, for a review).

The idea that supersonic turbulence may be important in the control of star formation has been recognised in recent years (see MacLow \& Klessen 2004, for a review). Turbulence - if dominant - can also rule the structure of the ISM, creating clouds and

\footnotetext{
* Appendices B-D are only available in electronic form at the CDS via anonymous ftp to cdsarc.u-strasbg.fr (130.79.128.5) or via
}

http: //cdsweb.u-strasbg.fr/cgi-bin/qcat? J/A+A/463/1227 cavities with regular properties of a fractal geometry, apparently similar to the structure formed via violent events. Structures produced by various processes are often characterised by the powerlaw parameter $\beta$ of the mechanical luminosity distribution of bubbles or holes. With a specific model this can be converted into a parameter describing the size distribution of these structures, then $\beta$ can be determined even for a medium with no stellar energy injection (e.g., a structure created by supersonic turbulence). Oey \& Clarke (1997) obtained $1.4 \leq \beta \leq 2.1$ for the nearby galaxies M 31, M 33, Holmberg II, SMC. Ehlerová \& Palouš (2005) deduced $\beta=1.6 \pm 0.3$ from the size distribution of HI shells in the outer Milky Way. Elmegreen (1999) derived $\beta=$ 2.15 for a medium with a structure of $D=2.3$ fractal dimension, also from the size distribution of holes. This structure should be representative of that created by supersonic turbulence.

The hot gas volume filling factor $f$ of a galaxy is also an important parameter in characterizing the life cycle of the interstellar medium. Although there are several estimates of $f$ based on theoretical considerations and semi-empirical models, the $f$ parameter of our Galaxy is hardly constrained observationally (Ferriére 1998; Gazol-Patiño \& Passot 1999). Recently Ehlerová $\&$ Palouš (2005) obtained $f=5 \%$ for the shell volume filling factor for the outer Galaxy through an automated identification of HI shells.

To derive these global parameters a large sample of object are needed, and it is not easy to construct such a database for our own Galaxy. Thilker et al. (1998) and Mashchenko et al. (1999) 
made efforts to detect HI shells automatically, based on a model of the shell. Recently Ehlerová et al. (2004) developed a modelindependent algorithm that automatically searches HI shells in data cubes and identified $\sim 1000$ structures in the LeidenDwingeloo Survey data. An artificial neural networks algorithm by Daigle et al. (2003) was successfully applied to the Canadian Galactic Plane Survey data. However, these works intended to detect HI holes based on the velocity information of the $21 \mathrm{~cm}$ line data rather than on morphology, therefore they cannot find bubbles at late stages of their evolution when the expansion has slowed down.

Shell- or arc-like intensity enhancements are reported in many tracers of the ISM (see Kiss et al. 2004, and references therein, for an introduction). The structure of the diffuse ISM is well-represented in the far-infrared, mainly observed as the Galactic cirrus emission (Low et al. 1984). Although some studies reported loop features identified on the far-infrared, these were either restricted to the Galactic midplane (Schwartz 1987) or performed for a special object type, e.g., Wolf-Rayet stars (Marston 1996). Most of the prominent HI loops are conspicuous in the far-infrared, too, due to their dust content, which is closely related to HI (Boulanger \& Perault 1988). Many individual loops of various origin were first identified on FIR images, e.g., the North Celestial Pole Loop (Meyerdierks 1991) is an example of a high velocity cloud - Galactic disc interaction. The Cepheus Bubble is a remarkable infrared loop (Kun et al. 1987), which is likely a combined result of multiple supernova explosions and stellar winds in the Cep OB 2 association.

In a recent work Kiss et al. (2004, hereafter KMT04) presented the results of a quest for far-infrared loop features in the 2nd Galactic Quadrant. They catalogued 145 loops and investigated their morphological and physical characteristics. This study was not restricted to the Galactic midplane, but reached high Galactic latitudes and was able to give a comprehensive view on the distribution of large scale intensity enhancements. In the present work - as a continuation of the KMT04 study - we extend the quest to the 1st, 3rd, and 4th Galactic Quadrants. These two works together provide the Catalogue of Far-InfraRed Loops in the Galaxy.

\section{Input data and data analysis}

We used the 60 and $100 \mu \mathrm{m}$ ISSA plates (IRAS Sky Survey Atlas, Wheelock et al. 1994) to explore the distribution of dust emission. Reprocessed IRAS $100 \mu \mathrm{m}$ and reddening maps by Schlegel et al. (1998) were investigated as well. We searched for loop- or arc-like features in these images in the $1^{\circ} \leq D \leq 40^{\circ}$ diameter range. The data reduction steps and derived parameters are the same as described in KMT04. For the detailed description of the data reduction and derived parameters we refer to KMT04 and Appendix A (online only) of this present paper.

\section{Results}

In our all-sky survey, 462 FIR loops have been identified. We found 317 loops in the 1st, 3rd, and 4th Galactic quadrants (presented in Appendix B, online only), beyond the 145 loops in KMT04 (2nd Galactic quadrant). The electronic version of the catalogue with additional data products can be found at: "http://kisag. konkoly.hu/CFIRLG".

The catalogue contains the name of the loop; the central Galactic coordinates, size, and position angle of the fitted ellipse; and other parameters describing the appearance of the loop in FIR images. For details, see Appendix B in the electronic version of the paper or Sect. 3 in KMT04. For a subsample of 43 loops of our catalogue (1st, 3rd, and 4th Galactic quadrants) we were able to derive distances, using the distances of associated objects. The selection criteria for distance indicators were the following:

i) objects related to possible energy injection sources (O/B stars, SN-remnants, etc.) had to be placed in the interior of the loop;

ii) density enhancements of the ISM (molecular/dark clouds, molecular cores, etc.) had to be in the interior or in the loop wall;

iii) there had to be at least two objects with similar distances;

iv) distances of individual objects had to agree within the uncertainties.

In many cases the distance of a loop has already been determined by previous studies. In Appendix $C$ we present the distances and a detailed list of distance estimators for these 43 loops. Figure 6 shows the distribution of estimated distances and the distances projected to the Galactic plane.

In most cases, however, no definite distance could be derived for a specific loop. In these cases we listed all possible associated objects (not taking into account the distances of the individual objects), which appear on the loop wall or in the interior. The list of these associated objects is presented in Appendix D.

\section{Discussion}

\subsection{Sky distribution}

The Galactic longitude distribution of the GIRLs partly reflect the spiral structure of the Galaxy (Figs. 2 and 3). There is a noticeable increase in loop counts towards the major spiral arms, i.e., the Local -, the inner Carina-Sagittarius -, and the outer Perseus Arms. The distribution of GIRLs in the sky is expected to reflect the exponential disc distribution of the ISM; accordingly, regions closed to the Galactic plane should be the most populated parts of sky in loops and this number should decrease nearly exponentially by the increasing Galactic latitude.

The distribution of loop centres in Galactic latitude (Fig. 4) which is expected to reflect the exponential disc distribution of the ISM - shows relatively large counts at high $|b|$ values, especially for outward locations. This cannot be explained by projection and distance effects only, in agreement with that was found for the 2nd Galactic quadrant (KMT04). To explain the formation of loops at high Galactic latitudes one needs an efficient process that does not necessarily have to take place close to the Galactic plane. This excludes SN-explosions and the stellar wind of massive stars as dominant effects. Although clouds infalling from the Galactic halo could create loops above the midplane, their infall rate is insufficiently small (Ehlerová \& Palouš 1996).

\subsection{Groups at larger scales}

The celestial distribution of the identified loops is rather complex, showing structures with scales even larger than the average loop diameters (Fig. 1). For example, two remarkable features forming "chains" of loops can be found around $l \approx 175^{\circ}, b \approx$ $+10^{\circ}$ and around $l \approx 115^{\circ}, b \approx-15^{\circ}$. This latter huge structure is well seen as a fragmented arc in HI, too, at velocities approximately $-40 \mathrm{~km} \mathrm{~s}^{-1}$ in the Leiden-Dwingeloo Survey (Hartmann $\&$ Burton 1997). Some groups of loop centres coincide well with the location of molecular complexes, including the Ophiucus 


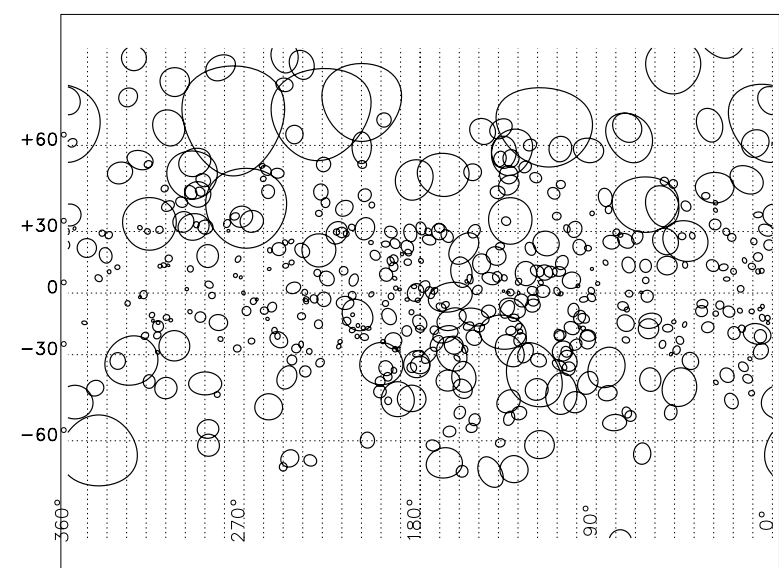

Fig. 1. Distribution of GIRLs in the sky (Galactic coordinate system, Mercator projection), represented by the fitted ellipses. Note that Mercator projection causes a size distortion at polar regions.

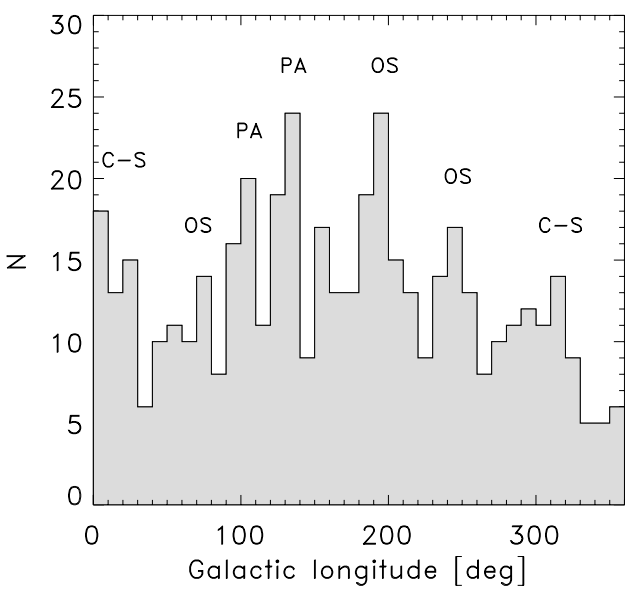

Fig. 2. Distribution of loops in Galactic longitude. The peaks in loopcount reflect the higher number density in the direction of Galactic arms (OS: Orion Spur, PA: Perseus Arm, C-S: Carina-Sagittarius Arm). The binsize is $10^{\circ}$.

Molecular Cloud $\left(l \approx 10^{\circ}, b \approx+15^{\circ}\right)$, the Ursa Maior Molecular Cloud $\left(l \approx 135^{\circ}, b \approx+55^{\circ}\right)$, the Taurus molecular complex $(l \approx$ $\left.180^{\circ}, b \approx+0^{\circ}\right)$, and the Orion-Monoceros molecular region $(l \approx$ $\left.210^{\circ}, b \approx-15^{\circ}\right)$. Although it is likely that loop structures are associated with large-scale molecular material, no other obvious occurrences were found on these scales in our sample.

\subsection{Dominated by confusion?}

To independently study the immediate inner and outer surrounding of the Solar System, we arbitrarily split the Galactic longitude space into two parts, approximately along the direction of the Local Arm, i.e., the $l=70^{\circ}-250^{\circ}$ axis. The loop counts in the inner and outer part show significant differences: there are almost twice as many loops in the outer region than in the inner one. One possibility to explain this fact is the existence of strong confusion in the direction of central (inward) Galactic regions, as discussed below (for the definition of Galactic regions A-D see Table 1).

The possibility of a confusion-effected distribution for the 2nd Galactic Quadrant was already mentioned in KMT04. In the present study - due to the extension to the whole sky - this
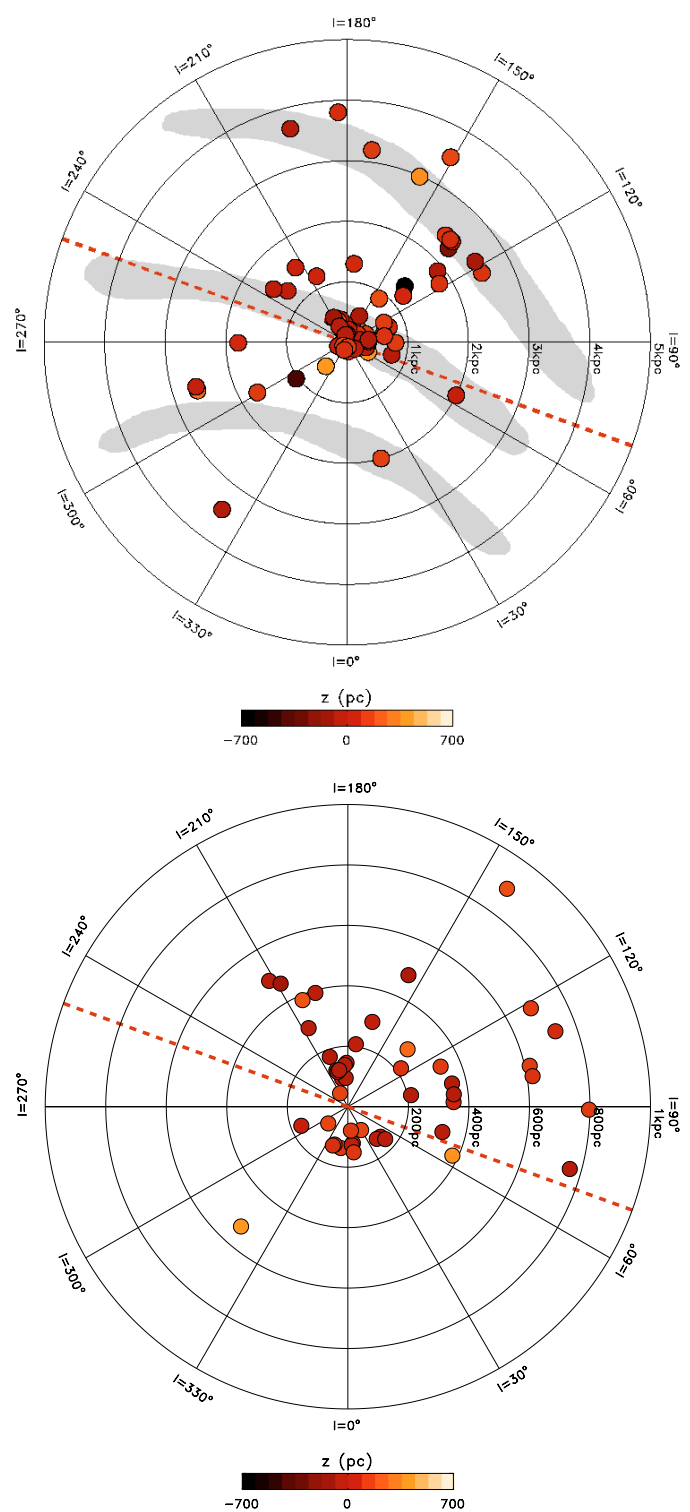

Fig. 3. Galactic distribution of loops with known distances around the Solar System (located at the panel centres). Top panel: location of infrared loops projected to the Galactic plane, up to a distance of $d=$ $5 \mathrm{kpc}$. Bottom panel: magnification of the central region $(d<1 \mathrm{kpc})$. The distance from the Galactic midplane is indicated by colours as given by the colour bars. The approximate position of Spiral Arms (grey areas) were adapted from Georgelin \& Georgelin (1976). The immediate outer surrounding of the Solar System was defined between $l=$ $70^{\circ}-250^{\circ}$, while the inner Galaxy space lies on the other side of the $l=$ $70^{\circ}-250^{\circ}$ axis (dashed red line, see also Table 1). The majority of the loops are probably located in the Local Arm, as explained in Sect. 4.4).

confusion effect is more expressed and manifests itself in two main ways:

i) As presented in Fig. 4, we see more loops in the "outer" Galaxy than in the "inner" regions for $|b|<30^{\circ}$ (region A), although probabilities for the frequency of high-pressure events (e.g., SN-rates) should be significantly higher in the inner Galaxy.

ii) The size distribution is different for regions $\mathrm{A}$ and $\mathrm{B}$ (see Fig. 5): in the inner Galaxy (A) the average apparent size is smaller than in B (4.1 and 5.2 , respectively). This discrepancy is not observed for higher Galactic latitudes (C and D). 
Table 1. Definition of four Galactic regions. Regions have been defined to help the comparison of loop characteristics at different parts of the sky.

\begin{tabular}{lcc}
\hline \hline Region & Galactic longitude & Galactic latitude \\
\hline A & $l<70^{\circ}$ or $l>250^{\circ}$ & $|b|<30^{\circ}$ \\
& inner & low \\
B & $70^{\circ}<l<250^{\circ}$ & $|b|<30^{\circ}$ \\
& outer & low \\
C & $l<70^{\circ}$ or $l>250^{\circ}$ & $30^{\circ} \leq|b| \leq 60^{\circ}$ \\
& inner & high \\
D & $70^{\circ}<l<250^{\circ}$ & $30^{\circ} \leq|b| \leq 60^{\circ}$ \\
& outer & high \\
\hline
\end{tabular}
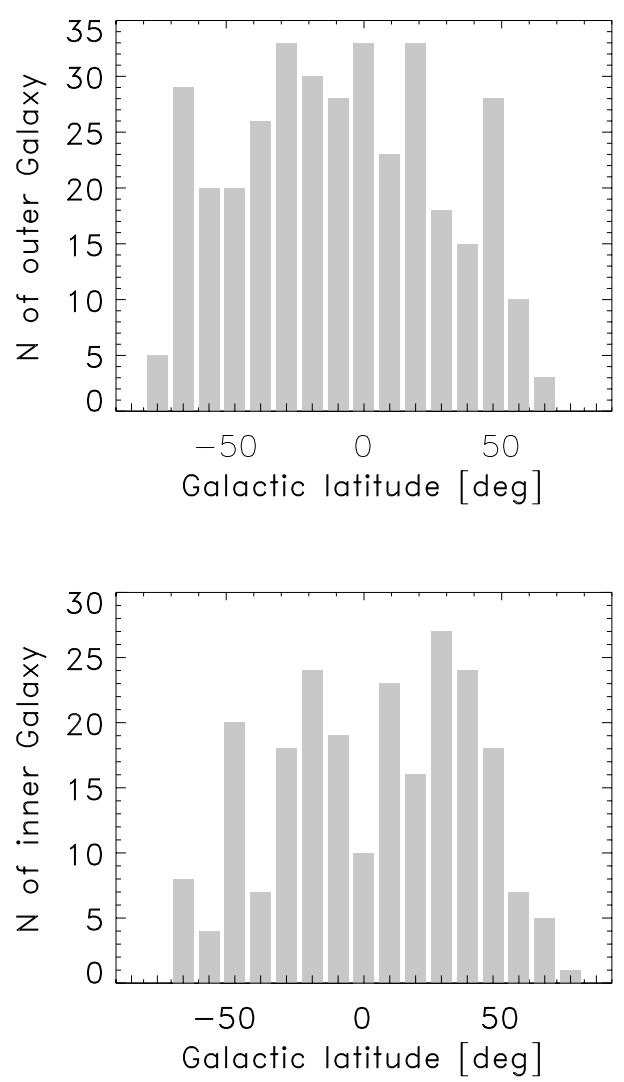

Fig. 4. Galactic latitude distribution of loops in the outer $(B+D$, top panel $)$ and inner $(\mathrm{A}+\mathrm{C}$, bottom panel $)$ Galaxy. See Table 1. The binsize is $10^{\circ}$.

These two facts together indicate that either (a) we miss large loops at low $|b|$ in the inner Galaxy due to the strong IR background (small loops are still visible since they are smaller than the characteristic scale of molecular clouds), or (b) large loops are really destroyed by frequent violent events and are only visible at their early evolutionary phases (i.e., at a small size). Unfortunately our present FIR data cannot distinguish between these possibilities.

\subsection{The large-scale distribution of ISM in the Galaxy}

In Appendix $\mathrm{C}$ we presented a subsample of 43 loops of our catalogue. For these loops we were able to derive distances using the distances of associated objects. Together with the 30-loop sample in Appendix B of KMT04, we have a sample of 73 loops with known distances. We used this sample to characterise the

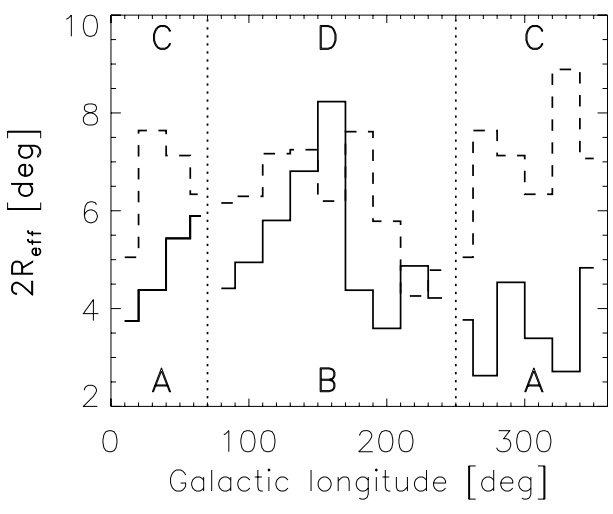

Fig. 5. Average effective loop diameter vs. Galactic longitude in the inner $(\mathrm{A}+\mathrm{C})$ and outer $(\mathrm{B}+\mathrm{D})$ Galaxy; solid and dashed lines correspond to $|b| \leq 30^{\circ}$ and $30^{\circ} \leq|b| \leq 60^{\circ}$, respectively. The binsize is $20^{\circ}$.
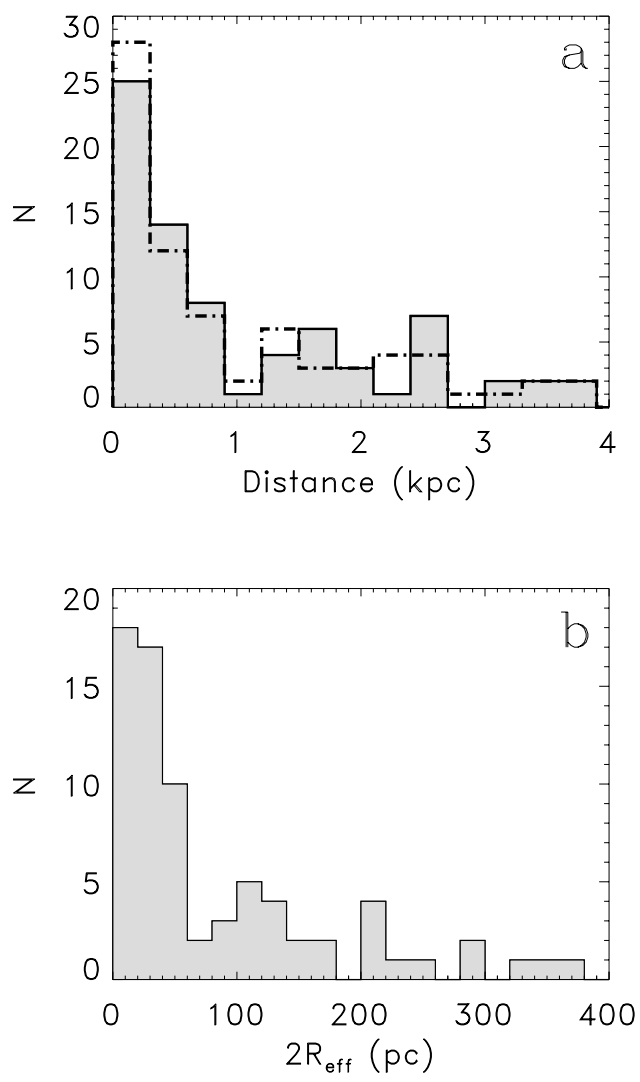

Fig. 6. a) Distribution of estimated distances for 73 loops. Dash-dotted line represents the distribution of distances projected to the Galactic plane. b) Distribution of effective diameters in the same sample. The widths of the bins are $300 \mathrm{pc}$ and $20 \mathrm{pc}$ for $\mathbf{a}), \mathbf{b}$ ), respectively.

large-scale distribution of the ISM in the vicinity of the Solar System.

\subsubsection{Size distribution of cavities}

In Fig. 7 we present the differences in distance distributions in the inward and outward Galactic regions. In the inward region A most of the loops are squeezed into a small area around the Solar System, within a distance of $\sim 0.2 \mathrm{kpc}$ (see Fig. 7). The rest of the loops do not show a concentration at a specific distance, but are rather randomly distributed in the interval $0.4 \mathrm{kpc} \leq d \leq 4 \mathrm{kpc}$. Note that the 2 nd Galactic quadrant has a major contribution to 

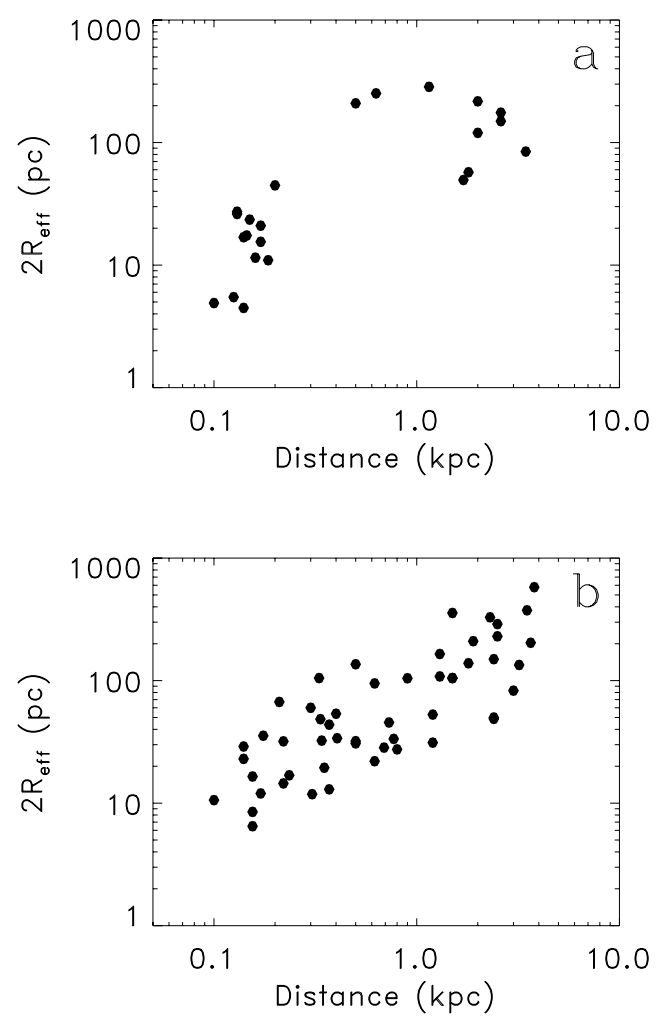

Fig. 7. Correlation of distance and effective size in the "inner" a) and in the "outer" Galaxy b), based on the sample of 73 loops with known distances.

outward regions, therefore this distribution is quite similar to the one found in KMT04. We checked the distances of the individual loops (those with known distances in regions A and B) and the distance of the wall of the Local Bubble in the direction of a specific loop using the maps and data based on the measurements of the NaI D-line doublet by Lallement et al. (2003). The reassuring result of this comparison was that all of these loops were in or behind the wall, i.e., behind the $W(\mathrm{D} 2)=20 \mathrm{~m} \AA$ contour of equivalent width (see Fig. 8).

The general structure of galaxies is often characterised by the distribution of bubbles and superbubbles, as was done for nearby galaxies by Oey \& Clarke (1997) and Kim et al. (2003). The generally used parameter $\beta$, the power-law index of the mechanical luminosity function, is closely related to the power-law index $s$ of the logarithmic size distribution: $s=2-2 \beta$ (see the model by Oey \& Clarke 1997). Using this relationship we derived a power-law index of $\beta=1.37 \pm 0.17$ for our outer sample (region B) for the interval $1.4 \leq \log \left(2 R_{\text {eff }}\right) \leq 2.6$, which is similar to the value of the 2 nd Galactic quadrant only $(\beta=1.24 \pm 0.30$, KMT04) and is in good agreement with $\beta=1.6 \pm 0.3$ obtained by Ehlerová \& Palouš (2005). Our value is lower than that of the other investigated galaxies (see Fig. 9b). The double-peaked distribution observed in KMT04 is less expressed in this larger sample and is closer to a power law. Due to the insufficient sample no proper $\beta$ could be derived for the inner Galaxy (region A), however, the distribution is very shallow (Fig. 9a).

We have no information on the distances of loops at high Galactic latitudes due to the lack of proper distance indicators. This prevented us from deriving proper $\beta$ values for high Galactic latitudes (C and D) as we did for A and B. However, the distribution of apparent size of the known-distance sample and that of the full high Galactic latitude sample are remarkably

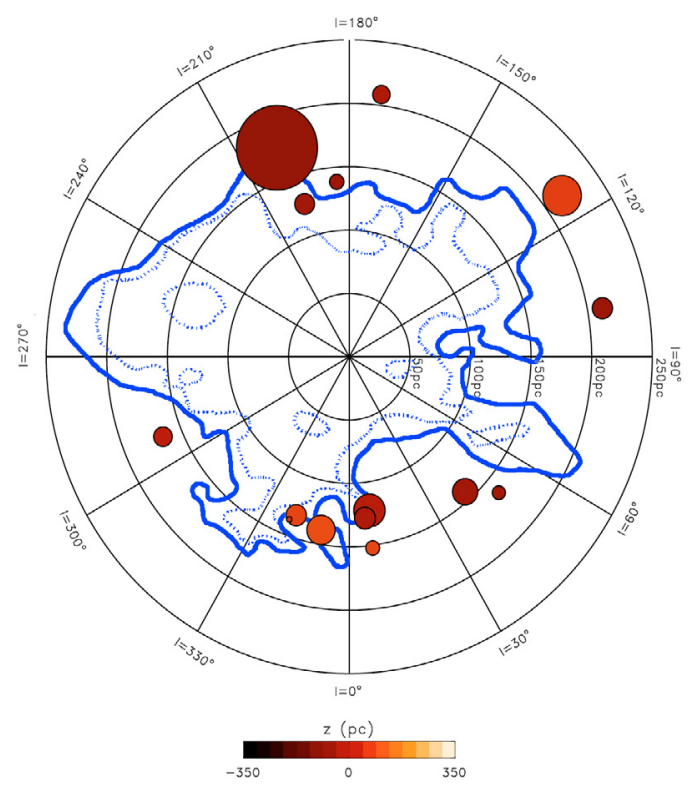

Fig. 8. Location of known distance loops in regions A and B in the close vicinity of the Solar System. The size of the filled circles indicates the real effective size of the loops. Dashed and solid blue curves correspond to the $W(\mathrm{D} 2)=20 \mathrm{~m} \AA$ and $W(\mathrm{D} 2)=50 \mathrm{~m} \AA$ contours of equivalent width of the NaI doublet line (see Lallement et al. 2003).

different. This indicates different real size distributions behind the apparent ones, and the presence of a different process governing the structure.

Due to the lack of distance information only the apparent size distribution can be used as diagnostic tool to test the structure of the high Galactic latitude ISM. In a simple model we tested the apparent size distribution with different $\beta$ values. The model included a real size distribution with a prescribed $\beta$ value (so that the slope of the $\log (R)$ vs. $\log (N[\log (R)])$ distribution is $s=2-2 \beta)$ and a certain distribution of cavity centres. We derived the apparent size distribution from this size/spatial location distribution. The apparent size distribution was then fitted by a power law (as in the case of the real size distribution), with a power-law index of $s_{\text {app }}$, in the interval $1^{\circ} \leq 2 R_{\text {app }} \leq 40^{\circ}$, i.e., in our investigated apparent diameter $\left(2 R_{\text {app }}\right)$ range.

The main features and results of this simple model were the following:

- Assuming a random, homogeneous distribution of cavity centres, with a minimal distance of $d_{\min }=0 \mathrm{pc}$, the final $s_{\text {app }}$ has little dependence on the absolute value of the maximal distance $d_{\max }$, as long as the apparent sizes are in the fitted range. Realistic estimates of the maximal distances are from $\sim 250 \mathrm{pc}$ (scale height of the Galactic disc; Nakanishi $\&$ Sofue 2003) to a few kpc (the maximal visibility distance), depending on the Galactic region we try to model.

- The assumption of $d_{\text {min }}=0$ is not realistic, since the Solar System is located inside the Local Bubble, and the loops must be behind its wall, as it is the case for the objects in our known-distance sample. We choose a minimal distance of $d_{\min }=130 \mathrm{pc}$ (a characteristic distance of the wall of the Local Bubble, see Lallement et al. 2003), while keeping the random, homogeneous distribution behind. In this configuration the apparent size distribution is clearly altered, compared to the $d_{\min }=0$ cases. The apparent size distribution is shallower, and the larger the maximal distance, the closer the $s_{\text {app }}$ values with $d_{\min } \neq 0$ to the ones with $d_{\min }=0$. Although 

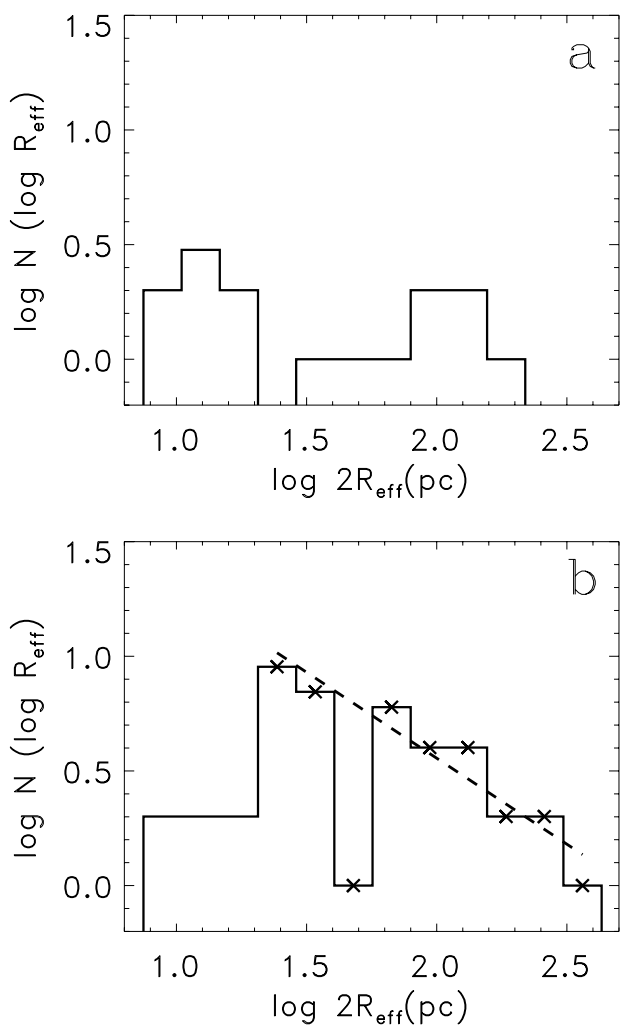

Fig. 9. Distribution of effective diameters for known-distance loops on a logarithmic scale in the "inner" a) and "outer" b) Galaxy. The relation of $\log 2 R_{\text {eff }}-\log N$ was fitted for $1.4 \leq \log \left(2 R_{\text {eff }}\right) \leq 2.6$. The points used for the fit are marked by asterisks (see Appendix B).

the difference is noticeable, $s_{\text {app }}$ has a stronger dependence on $\beta$ then on $d_{\min }$ (not considering very extreme conditions).

- We have checked with a Kolmogorov-Smirnov (KS) test, whether the apparent size distribution of high latitude loops in the northern and southern hemispheres may originate from the same distribution. The KS test resulted in a significance level of $\sim 50 \%$, showing that the two distributions are not really different. The $s_{\text {app,high }}$ values derived independently for the two hemispheres resulted in very similar values, therefore we used the data of the two hemispheres together in the following. There is a noticeable difference in the total counts (110 and 78 at the northern and southern hemispheres, respectively), but this does not affect the $s_{\text {app,high }}$ values.

- Assuming $\beta=1.37$ (that of the real size distribution in the known-distance sample), the apparent size distribution has $s_{\text {app }}=-0.7$ for the $d_{\text {min }}=0$, and $-0.7 \leq s_{\text {app }} \leq-0.5$ for the $d_{\text {min }}=130$ pc case, choosing the maximal distance in the range $250 \mathrm{pc} \leq d_{\max } \leq 1 \mathrm{kpc}$. This shows a good agreement with the power-law index of the apparent size distribution of the known-distance sample, $s_{\text {app,known }}=-0.67 \pm 0.12$.

- The known-distance sample is characteristic for low Galactic latitudes $\left(\mathrm{b} \leq 30^{\circ}\right)$ only. The power-law index of $s_{\text {app,high }}=$ $-1.78 \pm 0.21$ is quite different from $s_{\text {app,known }}$. The likely difference is a different real size distribution, i.e., a different $\beta$. We tested whether a realistic $\beta$ can preproduce this value: $\beta=2.15$ ( $s=-2.3$, the canonical value for a fractal structure created by supersonic turbulence) resulted in $s_{\text {app }}=-1.8$ for the $d_{\text {min }}=0$ and $-2.2 \leq s_{\text {app }} \leq-1.8$ for the $d_{\text {min }}=130 \mathrm{pc}$ case. These values are indeed very similar to $s_{\text {app,high }}$.

- We checked whether a size distribution with $\beta=1.37$ may be responsible for an apparent size distribution of $s_{\text {app }}=-1.78$, assuming a specific spatial distribution of cavity centres. To reproduce the required values of both parameters at the same time, the spatial distribution has to be such that the loops are highly concentrated at the outer edge of the investigated volume and practically no loops are found at small distances. This is an unlikely configuration even taking into account the fact that the Solar System is located inside the Local Bubble.

As we saw, various $\beta$ values lead to significantly different apparent size distributions, while changing the minimal and maximal distances in a realistic interval alters the size distribution less. Therefore it is not very likely that very different $\beta$ values would lead to the same $s_{\text {app }}$ using different spatial distributions, in the framework of our model. We have to note that among other limitations such as the unknown real spatial distribution, our simple model does not take into account any observational biases, which are especially important at small apparent sizes.

However, our simple model clearly outlines the difference in the real size distributions at low and high Galactic latitudes. A probable explanation for this difference is that the structure is governed by different physical processes. Our high latitude apparent size distribution favours a $\beta$ value similar to that of supersonic turbulence (Elmegreen 1999), which has been long considered as the most likely process forming the structure of the interstellar medium further from the Galactic midplane.

\subsubsection{Volume filling factor}

The hot gas volume filling factor $f$ is predicted to change with the Galactocentric distance (Ferriére 1998; Gazol-Patiño \& Passot 1999): it is $20 . . .30 \%$ for the inner Galaxy and decreases significantly for larger Galactocentric distances. For low Galactic latitudes $\left(b \leq 30^{\circ}\right)$ - where the structure is mostly formed by violent events - one can assume that the distribution of hot gas is very near to the distribution of holes/cavities and therefore can be characterised by their distribution. Applying the same procedure as in KMT04 (see Sect. 4 in KMT04, paragraph "Volume filling factor") for the whole loop sample in the inward and outward regions separately for $b \leq 30^{\circ}$ (regions A and B), we obtained $f_{\text {in }} \approx 30 \%$ and $f_{\text {out }} \approx 5 \%$ for $\mathrm{A}$ and $\mathrm{B}$, respectively. This latter value is very similar to the one presented in KMT04 for the 2nd Galactic quadrant $\left(4.6 \% \leq f_{\mathrm{KMT} 04}<6.4 \%\right)$. The size distribution in the inward region A almost completely misses the large loops, which might be due to confusion. However, they may not exist at all, since large structures are regularly destroyed by violent events. The $f_{\text {in }}$ value we found is of the order of $\sim 20 \%$, as predicted by Ferriére (1998) and Gazol-Patiño \& Passot (1999). Our $f_{\text {out }}$ value is very similar to $f_{\mathrm{HI}} \approx 5 \%$ found by Ehlerová \& Palouš (2005) through an automated identification of HI shells in the 2 nd Galactic quadrant.

\section{Summary}

We performed a survey of loop-/arc-like intensity enhancements in the diffuse far-infrared emission in the Galactic longitude intervals $0^{\circ} \leq l \leq 90^{\circ}$ and $180^{\circ} \leq l \leq 360^{\circ}$. Merged with the results of the second Galactic quadrant (KMT04), altogether we identified and catalogued 462 of these features. The Catalogue of Far-Infrared Loops in the Galaxy contains the basic physical properties and a list of associated objects as well. The electronic version of the catalogue is available at: http: //kisag. konkoly.hu/CFIRLG/. We also gave distance estimates for 73 loops based on the distance of associated objects. Our database provides a great opportunity to study the 
large-scale structure of the ISM in the Galactic neighbourhood of the Sun. We determined observational estimates for the hot gas volume filling factor of the the inner $\left(f_{\text {in }}\right)$ and outer $\left(f_{\text {out }}\right)$ Galactic environment of the Solar System and obtained $f_{\text {in }} \approx$ $30 \%$ and $f_{\text {out }} \approx 5 \%$. These values are in good agreement with both theoretical models and values derived from measurements in the radio domain. A study of the cavity size distribution has shown that the structure of the ISM is different at low and high Galactic latitudes. While at low latitudes it can be explained by high pressure events; the apparent size distribution of holes favours a fractal structure at high latitudes, similar to what can be created by supersonic turbulence.

Acknowledgements. We are grateful to L. G. Balázs and A. Pál for their kind help in the data analysis. This work was partly supported by the grants T043773 and K62304 of the Hungarian Scientific Research Fund (OTKA). We are indebted to our referee, Dr. Soňa Ehlerová, for her useful comments, which helped us to improve the paper.

\section{Appendix A: Detailed description of data reduction steps and main parameters}

- Identification of the loop structure: we investigated the 60 and $100 \mu \mathrm{m}$ ISSA plates (IRAS Sky Survey Atlas, Wheelock et al. 1994) to explore the distribution of dust emission. We created composite images of the $12.5 \times 12^{\circ} .5$ sized individual ISSA plates using the "geom" and "mosaic" procedures of the IPAC-Skyview package ${ }^{1}$, both at 60 and $100 \mu \mathrm{m}$. These images were built up typically from $10 \ldots 15$ ISSA plates, reaching a size of $\sim 40^{\circ} \times 40^{\circ}$. Loop-like intensity enhancements were searched for by eye on the $100 \mu \mathrm{m}$ mosaic maps. Loops by our definition must show an excess FIR intensity confined to an arc-like feature, at least $60 \%$ of a complete ellipse-shaped ring. A loop may consist of a set of bright, more or less isolated, extended spots, or may be a diffuse ring or part of a ring. The size of the mosaic image limits the maximal size of the objects found. On the other hand, due to the relatively large size of the investigated regions, loop-like intensity enhancements with a size of $\leq 1^{\circ}$ were not searched for. The original ISSA $I_{60}^{\mathrm{ISSA}}$ and $I_{100}^{\mathrm{ISSA}}$ surface brightness values were transformed to the COBE/DIRBE photometric system, using the conversion coefficients provided by Wheelock et al. (1994):

$-I_{60}=0.87 \times I_{60}^{\mathrm{ISSA}}+0.13 \mathrm{MJysr}^{-1}$

- $I_{100}=0.72 \times I_{100}^{\mathrm{ISSA}}-1.47 \mathrm{MJysr}^{-1}$.

Dust IR emission maps by Schlegel et al. (1998) (SFD) were investigated to derive parameters describing our loop features (see Sect. 2.2). The main differences of the SFD $100 \mu \mathrm{m}$ map with the ISSA maps are the following: (1) Fourier-destriping was applied; (2) asteroids and nonGaussian noise were removed; (3) IRAS and DIRBE $100 \mu \mathrm{m}$ maps were combined, preserving the DIRBE zero point and calibration; (4) stars and galaxies were removed.

We analysed the radial surface brightness profiles of the loops on the SFD $100 \mu \mathrm{m}$ map to check the effect of the removal of the sources mentioned above. We also used the SFD $E(B-V)$ maps derived from the dust column density maps. In the case of these maps the colour temperature was derived from the DIRBE 100 and $240 \mu \mathrm{m}$ maps, and a temperaturecorrected map was used to convert the $100 \mu \mathrm{m}$ cirrus map to a map proportional to dust column density (see SFD).

- Ellipse fitting: the shape of a loop candidate was approximated by an ellipse, which was then fitted using a 2D

${ }^{1}$ http://www.ipac.caltech.edu/Skyview/
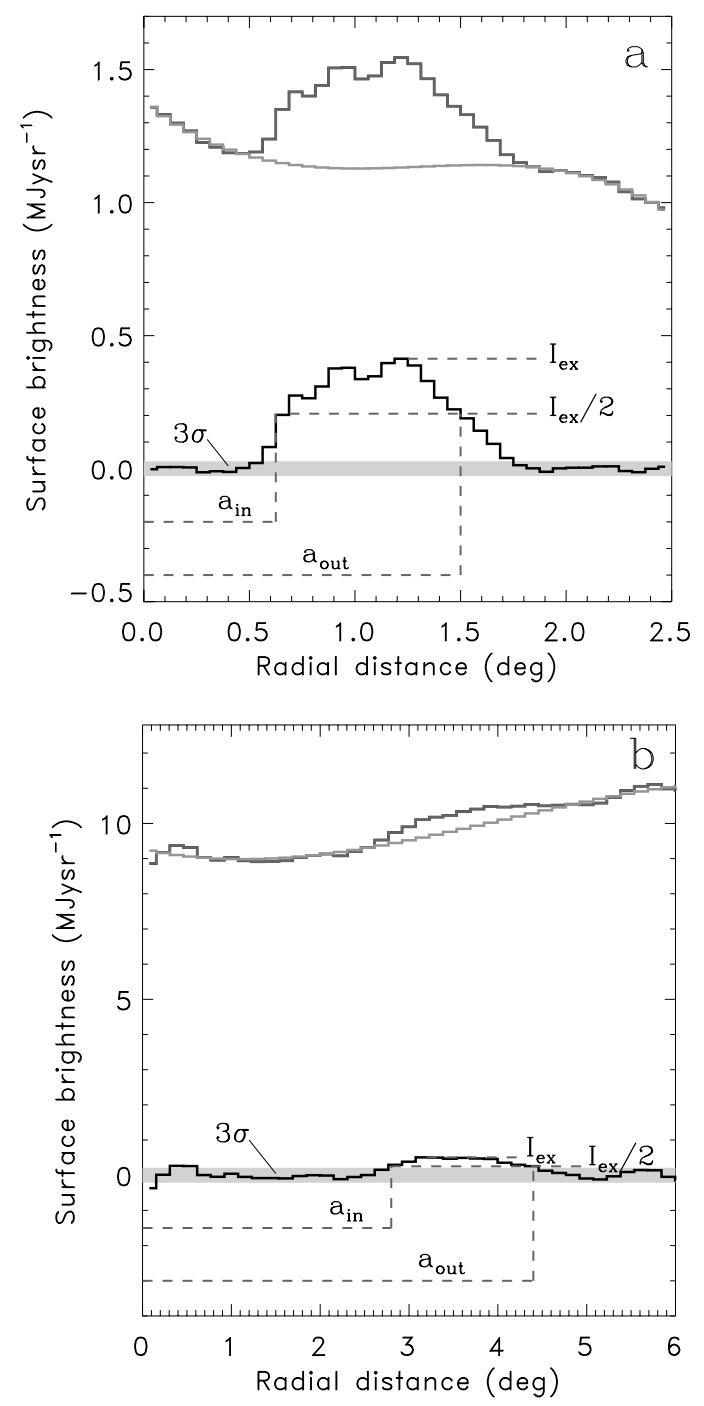

Fig. A.1. Example of an a) high significance loop $\left(\mathrm{G} 077-77, \Psi_{\mathrm{I} 100}=\right.$ $38.5)$ and a b) low significance loop $\left(\mathrm{G} 019-19, \Psi_{\mathrm{I} 100}=3.7\right)$. Besides low surface brightness enhancements, bright spot-like objects and point sources in the neighbourhood of the loop may also result in poor significance values, as was demonstrated in KMT04. Figures show the intensity profile before (top) and after (bottom) background removal (grey solid line), with the main derived parameters $\left(I_{\mathrm{ex}}, \sigma_{\mathrm{ex}}, a_{\mathrm{in}}, a_{\mathrm{out}}\right)$.

least-squares fit method. The fitted ellipse is defined with the central (Galactic) coordinates, the minor and major semiaxis of the ellipse, and the position angle of the major axis to the circle of Galactic latitude at the centre of the ellipse. This latter was defined to be "+" from East to North (or counter-clockwise).

- Intensity profile: for all of our loops we extracted radially averaged surface brightness profiles, extending to a distance of twice the major (and minor) axis of the fitted ellipse, using 40 concentric ellipsoidal rings. These surface-brightness profiles (ISSA 100 and $60 \mu \mathrm{m}$, SFD $100 \mu \mathrm{m}$ and SFD reddening maps) were used in the following to determine the basic parameters of the FIR emission in the loop; two examples are presented in Fig. A.1.

- Parameters of the loop wall: a local background was determined using the "non-loop" points in the radial surface brightness profiles, fitting a 3rd order polynomial, as demonstrated in Fig. A.1. This appropriate background was then removed from each surface brightness profile point. The 
intensity excess $I_{\mathrm{ex}}$ was derived as the maximum value of this background-removed profile. Inner and outer edges of the loop wall along the major axis $a_{\text {in }}$ and $a_{\text {out }}$, respectively, are defined as the radial distance at the full width at $I_{\mathrm{ex}} / 2$, the half power of the background-removed intensity profile. We distinguish three regions for a specific loop: (1) loop interior $\left(a<a_{\text {in }}\right)$; (2) loop wall ( $\left.a_{\text {in }} \leq a \leq a_{\text {out }}\right)$; (3) outer region $\left(a>a_{\text {out }}\right)$. The relative width of the wall of the fitted ellipse is defined as $W=1-a_{\text {in }} / a_{\text {out }}$.

- Significance: we calculated the standard deviation of the background-removed surface brightness, $\sigma_{\mathrm{ex}}$, in the "nonloop" positions ( $a \leq a_{\text {in }}$ and $a \geq a_{\text {aout }}$ ), and defined the significance of the loop as $\Psi=I_{\mathrm{ex}} / \sigma_{\mathrm{ex}}$. We derived significance parameters on 100 and $60 \mu \mathrm{m}$ ISSA maps and on the SFD $100 \mu \mathrm{m}$ point source removed sky brightness and reddening maps ( $\Psi_{\mathrm{I} 100}, \Psi_{\mathrm{I} 60}, \Psi_{\mathrm{S} 100}$, and $\Psi_{\mathrm{SEBV}}$, respectively). The higher the value of $\Psi$, the higher the intensity excess of the loop over the background, i.e., $\Psi$ can be used as a quality indicator. It was a requirement that a loop in the catalogue must show $\Psi \geq 3$ in at least one of the $\Psi$ values. Examples of the intensity profiles of high and low significance loops are shown in Fig. A.1.

- Colour index: $\Delta I_{60} / \Delta I_{100}$ colour indices were calculated for our loops from the radially averaged 60 and $100 \mu \mathrm{m}$ surface brightness profile. The colour index is defined as the slope of the $I_{60}$ versus $I_{100}$ scatter plot, using the data points of the surface brightness profiles in the positions of the loop wall only ( $a_{\text {in }} \leq a \leq a_{\text {out }}$ ). The uncertainties of $\Delta I_{60} / \Delta I_{100}$ given in the catalogue are the formal errors of the slope fitting.

- Associated objects: we attributed associated objects to our identified loops with the condition that an associated object has to be placed in the wall or in the interior of the loop. We considered the following type of possible associated objects (references are indicated):

- dark clouds (Dutra \& Bica 2002);

- supernova remnants (Green 1994);

- OB-associations (Lang 1992);

- pulsars (Taylor et al. 1993);

- HII regions (Sharpless 1959);

- IRAS point source with molecular core FIR colours;

- IRAS point source with T Tau star-like FIR colours.

Selection criteria for molecular cores and T Tauri stars were selected from the IRAS Point Source Catalogue (Joint IRAS Science Working Group 1988) according to the following criteria:

- point sources associated with galaxies were excluded;

- photometric qualities are 2 or better at 12, 25, and $60 \mu \mathrm{m}$;

- molecular cores: $0.4 \leq \log _{10}\left(\frac{F_{25}}{F_{12}}\right) \leq 1.0 \& 0.4 \leq$ $\log _{10}\left(\frac{F_{60}}{F_{25}}\right) \leq 1.3$

- $\mathrm{T}$ Tauri stars: $0.0 \leq \log _{10}\left(\frac{F_{25}}{F_{12}}\right) \leq 0.5 \&-0.2 \leq$ $\log _{10}\left(\frac{F_{60}}{F_{25}}\right) \leq 0.4$

following the definitions by Emerson (1998). $F_{12}, F_{25}$, and $F_{60}$ are the 12,25 , and $60 \mu \mathrm{m}$ uncorrected IRAS fluxes, respectively.

\section{References}

Abergel, A., Teyssier, D., \& Bernard, J. P. 2003, A\&A, 410, 577 Benson, P. J., \& Myers, P. C. 1989, ApJS, 71, 89

Blaauw, A. 1991, OB associations and the fossil record of star formation, in The Physics of Star Formation and Early Stellar Evolution, ed. C. J. Lada, \& N. D. Kylafis (Kluwer), 125
Blitz, L., Fich, M., \& Stark, A. A. 1982, ApJS, 49, 183 (BFS)

Boulanger, F., \& Perault, M. 1988, ApJ, 330, 964

Brand, P. W. J. L., \& Zealey, W. J. 1975, A\&A, 38, 363

Chini, R. 1981, A\&A, 99, 346

Cox, D. P., \& Smith, B. W. 1974, ApJ, 189, 105

Daigle, A., Joncas, G., Parizeau, M., \& Miville-Deschênes, M.-A. 2003, PASP, 115,662

Desert, F.-X., Bazell, D., \& Blitz, L. 1990, ApJ, 355, 51

Dolan, C. J., \& Mathieu, R. D. 2001, AJ, 121, 2124

Dutra, C. M., \& Bica, E. 2002, A\&A, 383, 631

Ehlerová, S., \& Palouš, S. 1996, A\&A, 313, 478

Ehlerová, S., \& Palouš, S. 2005, A\&A, 437, 101

Ehlerová, S., Palouš, S., \& Wünsch, R. 2004, Ap\&SS, 289, 279

Elmegreen, B. G. 1999, Phases and Structures of Interstellar Gas, in Physics and Chemistry of the Interstellar Medium, ed. V. Ossenkopf et al., GCA-Verlag Herdecke, 77

Emerson, J. P. 1998, Formation and Evolution of Low Mass Stars, Nato Advanced Institute (ASI) Series C, 241 (Dordrecht: Kluver), 193

Erlykin, A. D., \& Wolfendale, A. W. 2003, J. Phys. G, 29, 641

Ferriére, K. 1998, ApJ, 503, 700

Fich, M., Blitz, L., \& Stark, A. A. 1989, ApJ, 342, 272 (FBS)

Franco, G. A. P. 1989, A\&A, 223, 313

Fukui, Y., Onishi, T., Abe, R., et al. 1999, PASJ, 51, 751

Gazol-Patiño, A., \& Passot, Th. 1999, ApJ, 518, 748

Georgelin, Y. M., \& Georgelin, Y. P. 1976, A\&A, 49, 57

Green, D. A. 1994, A Catalogue of Galactic Supernova Remnants, in Proc. of the IAU Coll. 145, Supernovae and Supernova Remnants, ed. R. McCray, \& Z. Wang (Cambridge University Press)

Grosso, N., Alves, J., Wood, K., Neuhäuser, R., et al. 2003, ApJ, 586, 296

Hartmann, L., \& Burton, W. B. 1997, Atlas of Galactic Neutral Hydrogen (Cambridge University Press)

Heiles, C. 1984, ApJS, 55, 585

Hu, E. M. 1981, ApJ, 248, 119

Humphreys, R. M. 1978, ApJS, 38, 309

Jenkins, E. B., \& Meloy, D. A. 1974, ApJ, 193, 121

Joint IRAS Science Working Group 1988, Infrared Astronomical Satellite Catalouges, The Point Source Catalogue, version 2.0, NASA RP-1190

Kim, S., Staveley-Smith, L., Dopita, M. A., et al. 2003, ApJS, 148, 473

Kiss, Cs., Moór, A., \& Tóth, L. V. 2004, A\&A, 418, 131 (KMT04)

Koo, B.-C., Heiles, C., \& Reach, W. T. 1992, ApJ, 390, 108

Kun, M., Balázs, L., \& Tóth, I. 1987, Ap\&SS, 134, 211

Lang, K. R. 1992, Astrophysical Data: Planets and Stars (Heidelberg: Springer)

Lallement, R., Welsh, B. Y., Vergely, J. L., Crifo, F., \& Sfeir, D. 2003, A\&A, 411,447

Launhardt, R., \& Henning, T. 1997, A\&A, 326, 329

Low, F., Beintema, D. A., Gautier, F. N., et al. 1984, ApJ, 278, 19

Magnani, L., Hartmann, D., \& Speck, B. G. 1996, ApJS, 106, 447

Marraco, H. G., \& Rydgren, A. E. 1981, AJ, 86, 62

Marston, A. P. 1996, AJ, 112, 2828

MacLow, M.-M., \& Klessen, R. S. 2004, Rev. Mod. Phys., 76, 125

Mashchenko, S. Y., Thilker, D. A, \& Braun, R. 1999, A\&A, 343, 352

Mattila, K., Winnberg, A., \& Grasshoff, M. 1979, A\&A, 78, 275

McKee, C. F., \& Ostriker, J. P. 1977, ApJ, 218, 148

Meyerdierks, H., Heithausen, A., \& Reif, K. 1991, A\&A, 245, 247

Minkowski, R. 1958, IAUS, 8, 1048

Nakanishi, H., \& Sofue, Y. 2003, PASJ, 55, 191

Oey, M. S., \& Clarke, C. J. 1997, MNRAS, 289, 570

Penprase, B. E. 1992, ApJS, 83, 273

Penprase, B. E. 1993, ApJS, 88, 433

Preite-Martinez, A. 1988, A\&AS, 76, 317

Rizzo, J. R., Morras, R., \& Arnal, E. M. 1998, MNRAS, 300, 497

Schlegel, D. J., Finkbeiner, D. P., \& Davis, M. 1998, ApJ, 500, 525 (SFD)

Schwartz, P. R. 1987, ApJ, 320, 258

Sharpless, S. 1959, ApJS, 4, 257

Tapia, M., Roth, M., Vázquez, R. A., \& Persi, P. 2004, RMxAC, 22, 73

Tatematsu, K., Fukui, Y., Landecker, T.L., Roger, R.S., 1990, A\&A 237, 189

Thilker, D. A., Braun, R., \& Wakterbos, R. A. M. 1998, A\&A, 332, 429

Taylor, J. H., Manchester, R. N., \& Lyne, A. G. 1993, ApJS, 88, 529

Welty, D. E., \& Fowler, J. R. 1992, ApJ, 393, 193

Wheelock, et al. 1994, IRAS Sky Survey Atlas Explanatory Supplement, JPL Publication 94-11 (Pasadena: JPL)

White, G. J. 1993, A\&A, 274, 33

Whittet, D. C. B., Prusti, T., Franco, G. A. P., et al. 1997, A\&A, 327, 1194

Yonekura, Y., Hayakawa, T., Mizuno, N., et al. 1999, in Proceedings of Star Formation 1999, Star Formation 1999, ed. T. Nakamoto, Nobeyama Radio Observatory, 82

de Zeeuw, P. T., Hoogerwerf, R., de Bruijne, J. H. J., Brown, A. G. A., \& Blaauw, A. 1999, AJ, 117, 354 